\title{
Cardiovascular System Part
}

National Cancer Institute

\section{Source}

National Cancer Institute. Cardiovascular System Part. NCI Thesaurus. Code C25762.

A term that refers to individual anatomic parts of the heart or the vessels. 